\title{
Polymorphisms in the thymidylate synthase and dihydropyrimidine dehydrogenase genes predict response and toxicity to capecitabine-raltitrexed in colorectal cancer
}

\author{
JOSEFA SALGADO ${ }^{1}$, NATALIA ZABALEGUI ${ }^{4}$, CARMEN GIL $^{1}$, IGNACIO MONREAL $^{2}$, \\ JAVIER RODRÍGUEZ ${ }^{3}$ and JESÚS GARCÍA-FONCILLAS ${ }^{1,3}$
}

\author{
Laboratories of ${ }^{1}$ Biotechnology, ${ }^{2}$ Biochemistry and ${ }^{3}$ Department of Oncology, University Clinic of Navarra (CUN), \\ Avda. Pio XII 36; ${ }^{4}$ Laboratory of Gene Therapy Application, Centre for Applied Medical \\ Research (CIMA), Avda. Pio XII 55, 31008 Pamplona, Navarra, Spain
}

Received July 24, 2006; Accepted October 12, 2006

\begin{abstract}
Pharmacogenetics is an increasingly useful field where the genetic studies are becoming an important tool for predicting drug toxicity and/or efficacy. Thymidylate synthase (TS) and dihydropyrimidine dehydrogenase (DPD) gene polymorphisms could be highly informative tools in the clinical handling of colorectal cancer patients, who are following fluoropyrimidine based chemotherapy. Fifty-eight patients, with non-resectable metastatic colorectal cancer, were treated with capecitabine and raltitrexed, every three weeks. Patients were divided in a good-response group (complete and partial response) and a poor-response group (stable and progression). A genotype panel TS-DPD was evaluated. Results show that TS genotype analysis clearly differentiates patients with a worst response to a 5-fluorouracil based chemotherapy. DPD genotype was shown to be highly informative for prediction of toxicity of the treatment. These polymorphisms could represent an accurate, rapid and effective determination panel, indicative of resistance and toxicity for patients undergoing fluoropyrimidine based treatment.
\end{abstract}

\section{Introduction}

Colorectal cancer is the fourth most common malignancy globally and the second leading cause of cancer death in Western countries. Treatment with 5-fluorouracil (5-FU) and calcium leucovorin ( $\mathrm{LV}$ ) has been the standard therapy for over a decade. Capecitabine, a tumor-activated fluoropyrimidine carbamate potently inhibits the enzyme thymidylate

Correspondence to: Dr Josefa Salgado Garrido, Laboratory of Biotechnology, University Clinic of Navarra, Avda. Pio XII 36, 31008 Pamplona, Navarra, Spain

E-mail: jsalgadog@unav.es

Key words: thymidylate synthase, dihydropyrimidine dehydrogenase, polymorphism, capecitabine, colorectal cancer synthase (TS). Raltitrexed, a water-soluble folate-based agent, is also a TS inhibitor. Two polymorphisms have been described in the TS gene, a 28-bp double or triple tandem repeat on the enhancer region of the gene promoter (TSER), and a 6-bp deletion at the 3'UTR region (1-4). On the other hand, $70-80 \%$ of the administered 5-FU is normally degraded in vivo by the enzyme dihydropyrimidine dehydrogenase (DPD) (Fig. 1). A point mutation in the invariant GT splice donor site flanking exon 14 , (IVS14 $+1 \mathrm{G} \rightarrow \mathrm{A})$, causes exon skipping and leads to an inactive DPD allele $(5,6)$. The aim of this study is to analyze TS and DPD polymorphisms and their association to a good/poor response and toxicity to 5-FU based chemotherapy.

\section{Materials and methods}

Patients. Fifty-eight patients with histological or cytological diagnosis of metastatic colorectal cancer were recruited for the study. At least one bidimensionally measurable indicator lesion, that had not been irradiated, was required. The patients were 18 years of age, or older, with a life expectancy of at least 3 months and each gave a written consent. Patients with CNS involvement of their disease, neurological or psychiatric disorders that could interfere with treatment compliance, significant cardiac disease or myocardial infarction within the previous 12 months, serious uncontrolled infections, malabsorption syndrome or patients lacking physical integrity of their upper gastrointestinal tract were excluded. Patients were also not enrolled if significant abnormalities in neutrophils $\left(<1.5 \times 10^{9} / 1\right)$, platelets $\left(<100 \times 10^{9} / 1\right)$, serum creatinine or serum bilirubin ( $>1.5 \mathrm{x}$ upper normal limit), ALT, AST, alkaline phosphatase ( $>2.5 \mathrm{x}$ upper normal limit) were detected. The study was conducted in concordance with the declaration of Helsinki and all current amendments. Capecitabine $\left(1000 \mathrm{mg} / \mathrm{m}^{2}\right)$ was administered (total dose of $2000 \mathrm{mg} / \mathrm{m}^{2} / \mathrm{d}$ ) during 14 days in 3-week cycles at approximately 12-h intervals and within $30 \mathrm{~min}$ of a meal. Raltitrexed was administered intravenously $\left(3 \mathrm{mg} / \mathrm{m}^{2}\right)$ in $15 \mathrm{~min}$ in the first day and every 3 weeks. Treatment was continued until the scheduled assessment at 8 months or until the development of progressive disease if recorded earlier. Treatment interruption, 


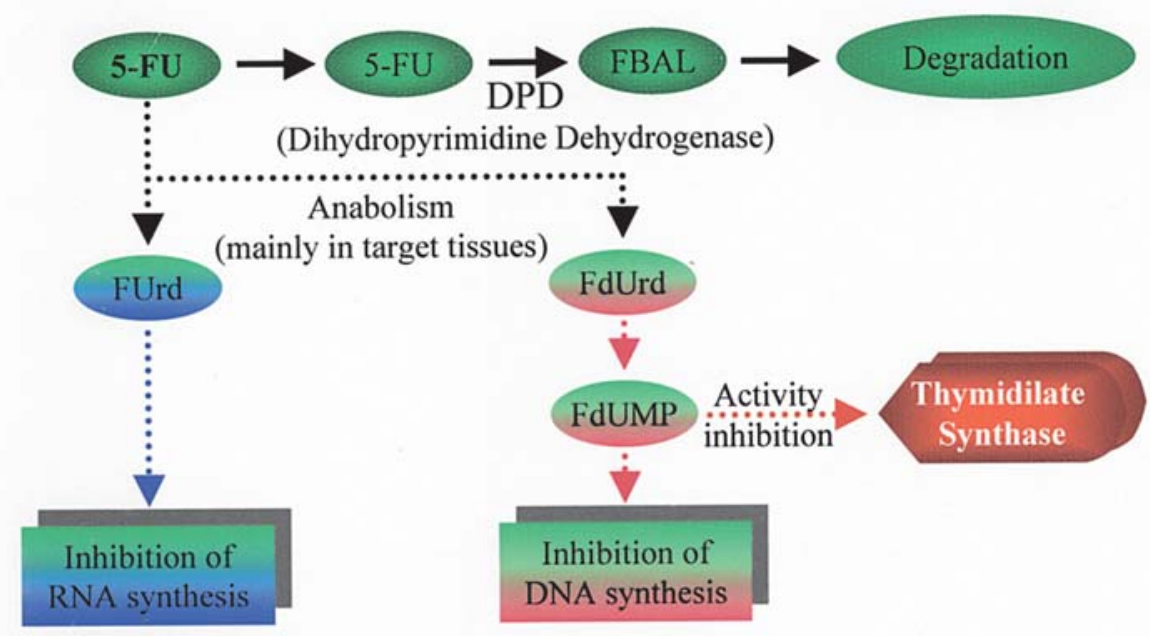

Figure 1. Pathways of 5-FU metabolism. FBAL, 5-fluoro-ß-alanine; FUrd, fluorouridine; FdUrd, fluorodeoxyuridine; FdUMP, fluorodeoxyuridine monophosphate.

or dose reduction, was not indicated for reactions unlikely to become serious or life-threatening and following the NCICCTC guidelines. Tumor response was assessed according to WHO criteria (7) and confirmed at least 4 weeks later by the same evaluation.

Genotyping. DNA was extracted from peripheral blood samples (MagNA Pure LC, Roche). TS polymorphisms were analyzed as described previously (8). Primers: TSER (forward) 5'-GTGGCTCCTGCGTTTCCCCC-3', TSER (reverse) 5'-GG CTCCGAGCCGGCCACAGGCATGGCGCGG-3', 3'UTR (forward) 5'-CAAATCTGAGGGAGCTGAGT-3' and 3'UTR (reverse) 5'-CAGATAAGTGGCAGTACAGA-3'. For the 3'UTR polymorphism, DraI was used as the restriction enzyme (8). Mutant DPD allele was detected by direct sequencing (9). Primers: DPD (forward) 5'-TCCTCTGCAA AAATGTGAGAAGGGACC-3' and DPD (reverse) 5'-TC
ACCAACTTATGCCAATTCTC-3'. A Genetic Analyzer ABI PRISM ${ }^{\circledR} 377$ (Applied Biosystems) was used.

Statistical analysis. TSER and 3'UTR polymorphisms were first analyzed separately. The $\chi^{2}$ test was used to compare the observed genotype distributions with those expected by the Hardy-Weinberg equilibrium. Secondary, $\chi^{2}$ or Fischer's twotailed exact test was used to determine the relationship between response to chemotherapy and TSER and 3'UTR genotypes of the TS gene. Finally, linkage disequilibrium between TSER and 3'UTR polymorphisms was also evaluated.

\section{Results}

Polymorphism at the TS enhancer region. Double or triple tandem repeats (28-bp) were named $2 \mathrm{R}$ (215 bp) and 3R (243 bp), respectively (Fig. 2A). A four tandem repeat (4R)

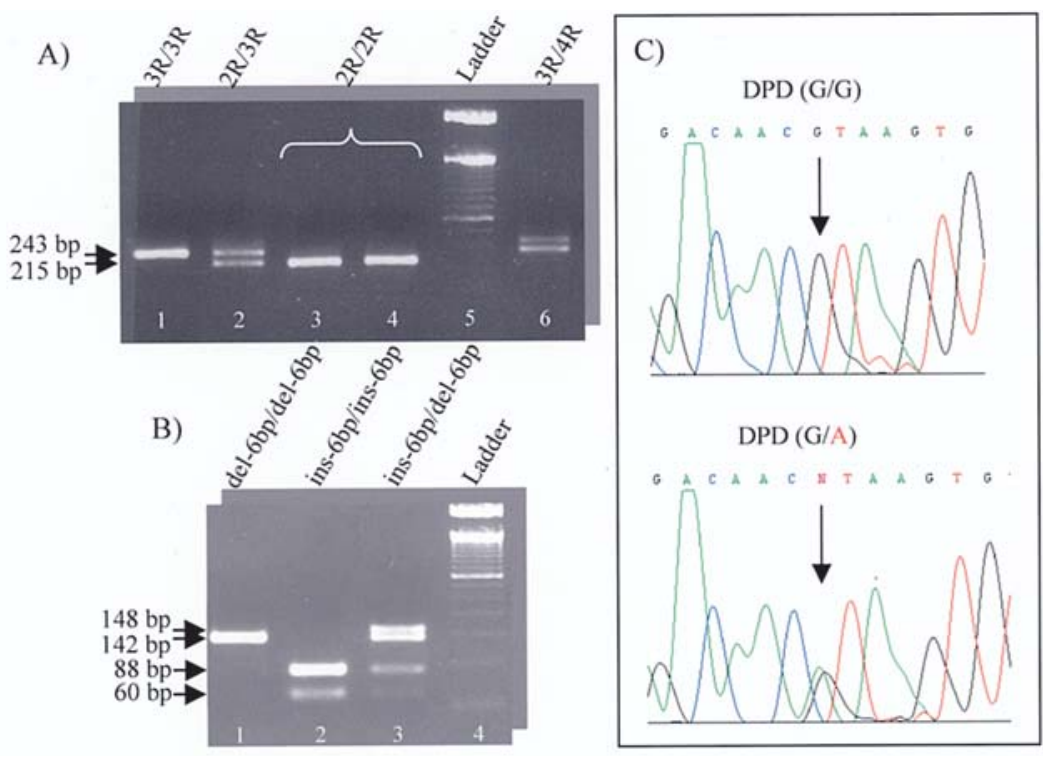

Figure 2. TS-DPD polymorphisms. (A), TSER polymorphisms (lane 5, 50 bp ladder); (B), 3'-UTR polymorphisms (lane 4, 50 bp ladder); (C), Direct sequencing of the DPD wild-type (G/G) and DPD mutant allele (G/A). 
Table I. Distribution of TS genotypes within the groups studied.

\begin{tabular}{lccc}
\hline & $\begin{array}{c}3 \mathrm{R} / 3 \mathrm{R} \\
(\%)\end{array}$ & $\begin{array}{c}2 \mathrm{R} / 3 \mathrm{R} \\
(\%)\end{array}$ & $\begin{array}{c}2 \mathrm{R} / 2 \mathrm{R} \\
(\%)\end{array}$ \\
\hline $\begin{array}{l}\text { Good-response } \\
\text { patients ( } \mathrm{n}=29)\end{array}$ & & & \\
(ins-6 bp/ins-6 bp) & 2 & 13 & 9 \\
(ins-6 bp/del-6 bp) & 5 & 20 & 4 \\
(del-6 bp/del-6 bp) & - & - & - \\
Poor-response & & & \\
patients (n=26) & & & \\
(ins-6 bp/ins-6 bp) & 4 & 4 & 2 \\
(ins-6 bp/del-6 bp) & 11 & 15 & - \\
(del-6 bp/del-6 bp) & 11 & - & - \\
\hline
\end{tabular}

-, no patients with these genotype combinations were found.

was observed in two samples (Fig. 2A, lane 6). Due to its low frequency, those samples were excluded from further statistical analysis. The frequencies were $15 \%(2 \mathrm{R} / 2 \mathrm{R}), 33 \%(3 \mathrm{R} / 3 \mathrm{R})$ and $52 \%(2 R / 3 R)$, in agreement with that predicted by the Hardy-Weinberg equilibrium, and in concordance with previous studies $(8,10-13)$.

Polymorphism at the TS 3'UTR. The genotype del-6 bp/del-6 bp shows a single fragment of $142 \mathrm{bp}$ whereas the ins- $6 \mathrm{bp} / \mathrm{ins}-$ 6 bp genotype is characterized by two fragments of 88 and 60 bp (Fig. 2B). The 148-bp band in the heterozygous sample is caused by incomplete digestion of the ins- 6 bp allele by the restriction enzyme. The distribution of the genotypes was $34 \%$ (ins-6 bp/ins-6 bp), 55\% (ins-6 bp/del-6 bp) and 11\% (del-6 bp/del-6 bp), which is in agreement with that predicted by the Hardy-Weinberg equilibrium. Previous studies obtained similar results $(3,8,13)$.

$D P D$ sequencing. Only one heterozygous subject for the IVS14+1G $\rightarrow$ A mutation was detected (Fig. 2C). The patient showed 5-FU high toxicity after the first cycle of treatment. Immediately, the 5-FU based chemotherapy was substituted by another more suitable treatment and the patient excluded from the study. The frequency of the heterozygote (1.7\%) was similar to previous studies (6).

Distribution of TS polymorphisms according to the overall objective tumor response. Patients were divided in a goodresponse group (complete and partial response) and in a poorresponse group (stable and progression). Frequencies of the TS polymorphisms are shown in Table I. The 3R/3R genotype is associated with the poor-response patients (26\%), being only $7 \%$ in the good response group; $2 \mathrm{R} / 2 \mathrm{R}$ genotype is increased $(13 \%)$ in the patients showing a good response to the treatment. The statistical analysis indicated that the $3 \mathrm{R} / 3 \mathrm{R}$ genotype was preferentially found within the poor-response group of patients $(\mathrm{p}<0.01)$ while the $2 \mathrm{R} / 2 \mathrm{R}$ genotype was more abundant in the good-response group. On the other hand, the ins-6bp/ins-6bp genotype was frequently present in the good-response group (24\%), while the double deletion (del-6 bp/del-6 bp) was only present in the patients with a poor-response $(\mathrm{p}<0.05)$. It has been reported that TSER and TS-3'UTR polymorphisms are in linkage disequilibrium (8). We have found that among individuals with 3R/3R genotype, there are differences of statistical significance $(p<0.05)$, being the genotype del- $6 \mathrm{bp} / \mathrm{del}-6 \mathrm{bp}$ segregated with the genotype $3 R / 3 R$.

\section{Discussion}

The aim of our study was to combine TS-DPD polymorphisms, that could predict response and toxicity to 5-FU based chemotherapy. Our results show that the $3 \mathrm{R} / 3 \mathrm{R}$ genotype is clearly associated with a poor response to the treatment. It has been reported that $3 R / 3 R$ is associated with a higher expression of mRNA than $2 \mathrm{R} / 2 \mathrm{R}(14,15)$. The principle that greater levels of TS translation could protect cells from the cytotoxic effect of 5-FU has been proposed as one mechanism for tumor resistance to this drug. But there is still controversy, since studies in colon cancer cell lines have found that the TSER genotype was related to the enzyme activity, but not to protein and mRNA levels (15). Additional studies are needed to identify the regulatory factors by which this polymorphism alters the TS gene expression and/or enzyme activity. We have detected two patients with the genotype 3R/4R. Although alleles containing 4, 5 and 9 copies of the tandem repeat have also been described previously, their effect remain unclear and its frequency low in the population (10). Regarding the TS-3'UTR polymorphism, the genotype del-6 bp/del-6 bp was only present in patients showing a poor response to the treatment. It would be possible that the deletion of $6 \mathrm{bp}$ in the 3'UTR region affects mRNA stability or secondary RNA structure, and could thus ultimately affect protein levels and/or regulation (3). In the context of the CRC, we have found that carrying 3R/3R and del-6 bp/del- 6 bp genotypes likely predicts a poor response to 5-FU based chemotherapy, being informative and helpful in individualizing patient treatment. Clearly, other genes could be implicated in the 5-FU resistance in CRC, since $30 \%$ of the patients with a poor response to the treatment do not carry $3 \mathrm{R} / 3 \mathrm{R}$ and/or del- $6 \mathrm{bp} /$ del-6 bp polymorphisms. Alterations of the cell through carcinogenesis could lead to various processes that would modify the response to the treatment. It has been published that TS polymorphisms are identical in normal and tumor tissues of homozygous individuals. However, in heterozygous samples, an imbalance between the $2 \mathrm{R}$ and $3 \mathrm{R}$ alleles in the tumor DNA was frequently observed, suggesting loss of heterozygosity $(\mathrm{LOH})$ at the TS locus $(16,17)$. This offers useful themes for undertaking larger prospective pharmacogenetic studies in the future. Regarding DPD deficiency, it is increasingly being recognized as an important pharmacogenetic factor in the aetiology of severe 5-FU associated toxicity $(6,18,19)$. Only one heterozygous subject for this mutation was detected, indicating a low prevalence of the mutation $(1.7 \%)$ in concordance with previous studies (6). Due to the high degree of 5-FU toxicity developed, the patient had to be excluded from the study. Considering the common use of fluoropyrimidines, genetic screening would be highly recommendable for the presence of the DPD gene 
mutation $(\mathrm{IVS} 14+1 \mathrm{G} \rightarrow \mathrm{A})$ related to toxicity, prior to $5-\mathrm{FU}$ administration.

In summary, our study is consistent with a model in which the TS-DPD polymorphism panel will give valuable tumor-response and toxicity information to greatly improve the selection and management of patients with CRC under a 5-FU based chemotherapy. Because only a blood sample from the patient is required, this analysis has become an easy, rapid and accurate determination in our center.

\section{Acknowledgements}

We thank members of the Department of Oncology at the Universitary Clinic of Navarra for their support. We are grateful to B. Honorato and F. García-Amigot at the Department of Biotechnology; and C. Mugueta at the Department of Biochemistry for critically reading and commenting on the article.

\section{References}

1. Horie N, Aiba H, Oguro K, et al: Functional analysis and DNA polymorphism of the tandemly repeated sequences in the 5'terminal regulatory region of the human gene for thymidylate synthase. Cell Struct Funct 20: 191-197, 1995.

2. Kawakami K, Salonga D, Park JM, et al: Different lengths of a polymorphic repeat sequence in the thymidylate synthase gene affects translational efficiency but not its gene expression. Clin Cancer Res 7: 4096-4101, 2001.

3. Ulrich CM, Bigler J, Velicer CM, et al: Searching expressed sequence tag databases: discovery and confirmation of a common polymorphism in the thymidylate synthase gene. Cancer Epidemiol Biomarkers Prev 9: 1381-1385, 2000.

4. Lenz H-J and Zhahedy S: A 6 base-pair deletion in the 3'UTR of the thymidylate synthase gene predicts TS mRNA expression in colorectal tumors. A possible candidate gene for colorectal cancer risk. Proc AACR 43: 660-667, 2002.

5. Wei X, McLeod HL, McMurrough J, et al: Molecular basis of the human dihydropyrimidine dehydrogenase deficiency and 5-fluorouracil toxicity. J Clin Invest 98: 610-615, 1996.

6. Van Kuilenburg AB, Muller EW, Haasjes J, et al: Lethal outcome of a patient with a complete dihydropyrimidine dehydrogenase (DPD) deficiency after administration of 5fluorouracil: frequency of the common IVS14+1G $>$ A mutation causing DPD deficiency. Clin Cancer Res 7: 1149-1153, 2001.
7. Bleiberg H, Rougier P and Wilke HJ: Colorectal cancer. WHO Handbook for reporting results of cancer treatment. World Health Organization Offset Publication. Martin Dunitz Ltd. 48, 1998

8. Ulrich CM, Bigler J, Bostick R, et al: Thymidylate synthase promoter polymorphism, interaction with folate intake, and risk of colorectal adenomas. Cancer Res 62: 3361-3364, 2002.

9. Van Kuilenburg AB, Haasjes J, Richel DJ, et al: Clinical implications of dihydropyrimidine dehydrogenase (DPD) deficiency in patients with severe 5-fluorouracil-associated toxicity: identification of new mutations in the DPD gene. Clin Cancer Res 6: 4705-4712, 2000.

10. Marsh S and McLeod HL: Thymidylate synthase pharmacogenetics in colorectal cancer. Clin Colorectal Cancer 1: 175-181, 2001.

11. Iacopetta B, Grieu F, Joseph D, et al: A polymorphism in the enhancer region of the thymidylate synthase promoter influences the survival of colorectal cancer patients treated with 5-fluorouracil. Br J Cancer 85: 827-830, 2001.

12. Marsh S, Collie-Duguid ES, Li T, et al: Ethnic variation in the thymidylate synthase enhancer region polymorphism among Caucasian and Asian populations. Genomics 58: 310-312, 1999.

13. Sharp L and Little J: Polymorphisms in genes involved in folate metabolism and colorectal neoplasia: a HuGE review. Am J Epidemiol 159: 423-443, 2004.

14. Marsh S, McKay JA, Cassidy J, et al: Polymorphism in the thymidylate synthase promoter enhancer region in colorectal cancer. Int J Oncol 19: 383-386, 2001.

15. Mauritz R, Beumer IJ, Marsh S, et al: Polymorphism of the thymidylate synthase gene and thymidylate synthase levels in colon cancer cell lines and different tissues of colorectal cancer patients. Nucleosides Nucleotides Nucleic Acids 23: 1381-1384, 2004.

16. Vogelstein B, Fearon ER, Kern SE, et al: Allelotype of colorectal carcinomas. Science 244: 207-211, 1989.

17. Kawakami K, Ishida Y, Danenberg KD, et al: Functional polymorphism of the thymidylate synthase gene in colorectal cancer accompanied by frequent loss of heterozygosity. Jpn J Cancer Res 93: 1221-1229, 2002.

18. Van Gennip AH, Abeling NG, Elzinga-Zoetekouw L, et al: Comparative study of thymine and uracil metabolism in healthy persons and in a patient with dihydropyrimidine dehydrogenase deficiency. Adv Exp Med Biol 253A: 111-118, 1989.

19. Etienne MC, Lagrange JL, Dassonville O, et al: Population study of dihydropyrimidine dehydrogenase in cancer patients. J Clin Oncol 12: 2248-2253, 1994. 